\title{
SEASONAL FOLATE SERUM CONCENTRATIONS AT DIFFERENT NUTRITION
}

\author{
Marica Krajčovičová-Kudláčková, Martina Valachovičová, Pavel Blažíček \\ Slovak Medical University, Bratislava, Slovak Republic
}

\section{SUMMARY}

Folic acid (vitamin B9) rich sources are leafy green vegetables, legumes, whole grains, egg yolk, liver, and citrus fruit. In winter and early spring, there could be insufficient supply of vegetables and fruit and thus lower intake of folic acid and possible deficient folic acid blood concentrations. The aim of the study was to assess serum vitamin B9 concentrations depending on the season (the last third of winter - March, the last third of spring - May/June and the beginning of autumn - September) and different nutritional habits (apparently healthy adults non-smoking, non-obese 366 subjects; 204 persons of general population on traditional mixed diet; and 162 long-term lacto-ovo vegetarians). In general population group, the mean concentration of folate in March was low (narrowly above lower reference limit) with high incidence of deficient values - 31.5\%. In May/ June vs. March was folate concentration significantly higher with deficient values in $13.2 \%$ of individuals. The highest serum values were observed in September with $11.1 \%$ of deficient values. In vegetarian vs. non-vegetarian group, significantly higher folate concentrations were found in each season with no deficient values. Folate and vitamin B12 are the regulators of homocysteinemia; plant food lacks of vitamin B12. The deficient folate serum values in March caused the mild hyperhomocysteinemia in $12.3 \%$ of individuals vs. only $5.9 \%$ and $4.8 \%$ of subjects in groups investigated in May/June and September. In spite of high folate concentrations in all investigations and no deficient value, $19.6-22.8 \%$ of vegetarians suffer from mild hyperhomocysteinemia as a consequence of deficient vitamin B12 concentrations in one quarter of subjects. As far as the general population is concerned, our findings suggest that winter and early spring are critical seasons in regards to optimal serum folate concentrations.

Key words: folate, vegetarians, general population, season

Address for correspondence: M. Kudláčková, Slovak Medical University, Limbova 12, 83303 Bratislava, Slovak Republic. E-mail: marica. kudlackova@szu.sk

\section{INTRODUCTION}

The term folate is used as a generic name for a group of compounds based on the folic acid structure. Folate or vitamin B9 is one of the 13 essential vitamins. They cannot be synthesized de novo by body and must be obtained from diet or supplementation. Neither folate nor folic acid is metabolically active. Both must be reduced to participate in cellular metabolism (1). Folic acid must be converted through dihydrofolate and tetrahydrofolate to the biologically active L-5-methyltetrahydrofolate. These conversions are necessary to provide one-carbon transfer reactions needed for purine/pyrimidine synthesis, for DNA methylation, and to regulate homocysteine ( $\mathrm{HCy}$ ) metabolism and optimal functionality of the methylation cycle (2).

Recent investigations have demonstrated beneficial effects of folates unrelated to homocysteine decrease (3) suggesting independent properties. One such mechanism could be free radical scavenging and antioxidant activity. Free radicals play an important role in the oxidative stress leading to many disorders and diseases. It was found that folic acid and, in particular, its reduced derivates act both directly and indirectly to produce antioxidative effects. Folates interact with the endothelial enzyme NO synthase and, exert effects on the cofactor bioavailability of $\mathrm{NO}$ and thus, on peroxynitrite formation.

The aim of this study was to assess seasonal serum folate concentrations depending on different nutritional habits. Rich sources of folic acid are leafy green vegetables, legumes, whole grains, citrus fruit, egg yolk, and liver (4). In winter and early spring, there can be insufficient intake of vegetables and fruit rich in folic acid and thus deficient folate blood concentrations can be recorded. Vitamin B12 and atherogenic homocysteine (HCy) were measured as additional values.

\section{MATERIALS AND METHODS}

Randomly selected apparently healthy adults, non-smoking, non-obese 366 subjects (165 men, 201 women) were divided into two groups depending on the nutritional habits, which were detected according to the data from standardized and validated questionnaires containing 146 food items. Nutritional group 1: 204 subjects (95 men, 109 women) of general population on traditional mixed diet, nutritional group 2: 162 long-term vegetarians (lacto-ovo, 70 men, 92 women, who consumed predominantly plant food with addition of dairy products and eggs). All subject lived in the same region (Bratislava and surroundings). The probands were investigated gradually - first part (127 subjects) in March (the last third of winter), second part (125 subjects) in May/June (the last third of spring) and other part (114 subjects) in September (the beginning of autumn). The group characteristic is showed in Table 1. The results were produced by realization of projects about health benefits and risks of alternative forms of nutrition vs. general population as the control group. The Regional Ethic Committee approved these studies, and all participants gave their written informed consent. 
Blood was sampled after an overnight fasting by a standard procedure. Serum folate and vitamin B12 concentrations were determined using Elecsys 2010 System (Roche Diagnostics, Basel, Switzerland). Plasma concentrations of total HCy were measured by high-performance liquid chromatographic method (HPLC) with fluorescence detection and SBD-F as a derivation agent (5). EDTA was used as an anticoagulant. Intake of folic acid, vitamin B12, other vitamins and mineral and trace elements only in natural form was considered (no supplementation). The probands consumed no folic acid fortified food. The quantitative data are presented as means \pm SEM. The significance of differences in measured values between groups was determined by unpaired Student's t-test; $\mathrm{p}$ values less than 0.05 were considered statistically significant.

\section{RESULTS}

In the general population group the mean concentration of folate in March was low (only $3.3 \mathrm{nmol} / \mathrm{l}$ above lower reference limit) with high incidence of deficient values $-31.5 \%$ (Table 1 ). In May/June vs. March the folate concentration was significantly higher with deficient values in $13.2 \%$ of individuals. The highest serum value was observed in September - only $11.1 \%$ of subject with deficient values. In vegetarian vs. non-vegetarian group significantly higher folate concentrations were found in each season (Table 1) with no deficient values. Significantly higher folate values in both nutritional groups were recorded in May/ June and September vs. March.

Vitamin B12 concentrations in vegetarians vs. non-vegetarians were significantly reduced with approximately one quarter of deficient values vs. no deficient subject in the general population group (Table 1). In subjects on traditional mixed diet the deficient folate serum values in March caused the increased HCy plasma concentrations in $12.3 \%$ of individuals vs. only $5.9 \%$ and $4.8 \%$ of subjects in groups investigated in May/June and September. In vegetarians the risk plasma $\mathrm{HCy}$ concentrations were found in $19.6-22.8 \%$ of subjects in all seasonal groups (Table 1).

\section{DISCUSSION}

One of many functions of folate and B12 vitamins as metabolic regulators is their presence in HCy metabolism. Vitamin B12 is absent in plant food. Vitamin deficiency is one of the risk factors of alternative nutrition. This deficiency can lead to several adverse health consequences: folate trapping in the methylation cycle and subsequent impaired DNA biosynthesis, pernicious anemia hematologically similar to that caused by folate deficiency, elevated atherogenic blood homocysteine and neural tube defects (6). Consumption of animal source food provides a better possibility to meet the needs for vitamin B12 (7).

HCy is a sulfur-containing amino acid requiring sufficient concentrations of folate and vitamin B12 for its degradation in

Table 1. Folate, vitamin B12 and homocysteine concentrations in groups of general population and vegetarians, prevalence of deficient vitamin values and hyperhomocysteinemia

\begin{tabular}{|c|c|c|c|}
\hline $\begin{array}{l}\text { Season } \\
\text { Months }\end{array}$ & $\begin{array}{l}\text { Last third of winter March } \\
\text { (first decade) }\end{array}$ & $\begin{array}{l}\text { Last third of spring May/June } \\
\text { (end/beginning) }\end{array}$ & $\begin{array}{l}\text { Beginning of autumn September } \\
\text { (third decade) }\end{array}$ \\
\hline \multicolumn{4}{|l|}{ General population } \\
\hline$n(m+w)$ & $73(33+40)$ & $68(32+36)$ & $63(30+33)$ \\
\hline Age range $(y)$ & $20-63$ & $20-66$ & $22-59$ \\
\hline Folate (nmol/l) & $12.8 \pm 0.4$ & $16.8 \pm 0.7^{\wedge}$ & $22.4 \pm 0.7^{\wedge}$ \\
\hline$<9.53$ & $31.5 \%$ & $13.2 \%$ & $11.1 \%$ \\
\hline Vitamin B12 (pmol/l) & $348 \pm 11$ & $318 \pm 10$ & $326 \pm 9$ \\
\hline$<179$ & $0 \%$ & $0 \%$ & $0 \%$ \\
\hline Homocysteine $(\mu \mathrm{mol} / \mathrm{l})$ & $13.4 \pm 0.5$ & $9.8 \pm 0.3^{\wedge}$ & $10.2 \pm 0.4^{\wedge}$ \\
\hline$>15$ & $12.3 \%$ & $5.9 \%$ & $4.8 \%$ \\
\hline \multicolumn{4}{|l|}{ Vegetarians } \\
\hline$n(m+w)$ & $54(25+29)$ & $57(23+34)$ & $51(22+29)$ \\
\hline Age range $(y)$ & $20-64$ & $20-67$ & $20-67$ \\
\hline Duration of vegetar. (y) & $12.4 \pm 0.6$ & $9.1 \pm 0.4$ & $9.6 \pm 0.5$ \\
\hline Folate (nmol/l) & $18.7 \pm 0.5^{*}$ & $30.6 \pm 0.6 * \wedge$ & $31.4 \pm 0.7 * \wedge$ \\
\hline$<9.53$ & $0 \%$ & $0 \%$ & $0 \%$ \\
\hline Vitamin B12 (pmol/l) & $245 \pm 8$ * & $238 \pm 6$ * & $246 \pm 9$ * \\
\hline$<179$ & $25.9 \%$ & $26.3 \%$ & $23.5 \%$ \\
\hline Homocysteine $(\mu \mathrm{mol} / \mathrm{l})$ & $14.5 \pm 0.3$ & $14.1 \pm 0.5^{*}$ & $13.9 \pm 0.4$ * \\
\hline$>15$ & $22.2 \%$ & $22.8 \%$ & $19.6 \%$ \\
\hline
\end{tabular}

${ }^{*} p<0.001$ (vegetarians vs. general population)

$\wedge p<0.001$ (May/June, September vs. March)

Reference values: folate 9.53-45.17 nmol//, vitamin B12 179-660 pmol// (9), lower limit for mild hyperhomocysteinemia $15 \mu \mathrm{mol} / \mathrm{l}$ (7). 
methylation cycle and maintenance of normal HCy values (8). In case of lower methionine intake (alternative nutrition, normal weight subjects of general population) prevails remethylation pathway of HCy metabolism (9). In spite of high folate concentrations in all investigations and no deficient value, approximately $20 \%$ of vegetarians suffer for mild hyperhomocysteinemia as a consequence of deficient vitamin B12 concentrations. In our previous study, we recorded $78 \%$ of deficient vitamin B12 serum values and $53 \%$ incidence of mild hyperhomocysteinemia in vegans - strict vegetarians (10). From vitamin B12 deficiency point of view and mild hyperhomocysteinemia prevention, vegetarians must be controlled and consume vitamin B12 fortified foods or take vitamin B12 supplements (11).

$\mathrm{HCy}$ was shown to be an independent risk factor for neurodegenerative and cardiovascular disease and it was long believed that the beneficial effects of folate and vitamin B12 on vascular function and disease are related directly to the HCy decrease $(12,13)$. Recent reports confirmed, as a collateral finding, that hyperhomocysteinemia is merely present in subjects suffering from acute or chronic cardiovascular events. Reduced methylation potential due to decreased S-adenosyl-methionine/S-adenosylhomocysteine ratio, induced by the elevated plasma HCy concentrations seems to be responsible for cardiovascular diseases (13). Other data suggest that risk values of HCy not only promote cellular and protein injury via oxidant mechanisms, but are also markers of the presence of pathological oxidative stress. Thus, it is possible that hyperhomocysteinemia is rather a marker of systemic or endothelial oxidative stress that is a major mediator of cardiovascular disorders (12). It has been suggested that hyperhomocysteinemia may promote the production of hydroxyl radicals which are the initiators of lipid peroxidation process. In spite of high incidence of hyperhomocysteinemia in vegetarians, the risk increased products of lipid peroxidation were found in $8 \%$ of subjects only (14) as a consequence of a better antioxidative status in comparison to subjects of general population.

Our findings suggest that the end of winter and early spring are critical seasons as regards provision of the recommended dietary allowance for folic acid. This fact results in deficient folate serum values in one third of the general population. The seasonal variations in blood folate concentrations were described in literature - significantly lower red cell and serum folate in spring compared with autumn or winter (15) as well as the prevalence of folate deficiency with significantly lower plasma folate concentrations in spring and lower erythrocyre folate concentrations in seasons other than summer (16). Attempts to increase the folic acid intake in the general population mainly in winter and early spring and to eliminate deficient serum values caused by insufficient rich folic acid food consumption include multivitamin supplementation and fortification of grain-based products. However, several reports suggest that folate has a procarcinogenic effect. Antifolates, such as methotrexate, are used in cancer treatment. Baggott et al. (17) analyzed the cancer incidence of six previously published large prospective folic acid supplementation trials. The analyses suggest that cancer incidence was higher in folic acid supplemented groups than the non supplemented groups (relative risk 1.21). Dietary supplementation with folic acid around the time of conception has long been known to reduce the risk of neural tube defects in the offspring (18). It is known for some time that folic acid daily intake $(400 \mu \mathrm{g}$ dose) is needed to optimize erythrocyte folic acid concentrations (19) and this dose was determined as recommended dietary allowance. The US National Institutes of Health and the Institute of Medicine have recommended $600 \mu \mathrm{g}$ of folic acid daily for pregnant women and $500 \mu \mathrm{g}$ during lactation (4). The potential risk of high dose folic acid supplementation must be considered. First, folate supplementation can mask vitamin B12 deficiency and second, literature data describe the potentially adverse effects of unmetabolized synthetic folic acid with regard to cancer, depression, and cognitive impairment $(20,21)$.

\section{REFERENCES}

1. Pietrzik K, Bailey L, Shane B. Folic acid and L-5-methyltetrahydrofolate: comparison of clinical pharmacokinetics and pharmacodynamics. Clin Pharmacokinet. 2010 Aug;49(8):535-48.

2. Suh JR, Herbig AK, Stover PJ. New perspectives on folate catabolism. Annu Rev Nutr. 2001;21:255-82.

3. Stanger O, Wonisch W. Enzymatic and non-enzymatic antioxidative effects of folic acid and its reduced derivates. Subcell Biochem. 2012;56:131-61.

4. Greenberg JA, Bell SJ, Guan Y, Yu YH. Folic Acid supplementation and pregnancy: more than just neural tube defect prevention. Rev Obstet Gynecol. 2011 Summer;4(2):52-9.

5. Vester B, Rasmussen K. High performance liquid chromatography method for rapid and accurate determination of homocysteine in plasma and serum. Eur J Clin Chem Clin Biochem. 1991 Sep;29(9):549-54.

6. Varela-Moreiras G, Murphy MM, Scott JM. Cobalamin, folic acid, and homocysteine. Nutr Rev. 2009 May;67 Suppl 1:S69-72.

7. Gilsing AM, Crowe FL, Lloyd-Wright Z, Sanders TA, Appleby PN, Allen $\mathrm{NE}$, et al. Serum concentrations of vitamin B12 and folate in British male omnivores, vegetarians and vegans: results from a cross-sectional analysis of the EPIC-Oxford cohort study. Eur J Clin Nutr. 2010 Sep;64(9):933-9.

8. Rasmussen K, Møller J. Total homocysteine measurement in clinical practice. Ann Clin Biochem. 2000 Sep;37( Pt 5):627-48.

9. Krajcovicova-Kudlackova M, Blazicek P, Mislanova C, Valachovicova M, Paukova V, Spustova V. Nutritional determinants of plasma homocysteine. Bratisl Lek Listy. 2007;108(12):510-5.

10. Krajčovičová-Kudláčková M, Blažíček P, Kopčová J, Béderová A, Babinská K. Homocysteine levels in vegetarians versus omnivores. Ann Nutr Metab. 2000;44(3):135-8.

11. Elmadfa I, Singer I. Vitamin B-12 and homocysteine status among vegetarians: a global perspective. Am J Clin Nutr. 2009 May;89(5):1693S$1698 \mathrm{~S}$.

12. Hoffman M. Hypothesis: hyperhomocysteinemia is an indicator of oxidant stress. Med Hypotheses. 2011 Dec;77(6):1088-93.

13. Cacciapuoti F. Hyper-homocysteinemia: a novel risk factor or a powerful marker for cardiovascular diseases? Pathogenetic and therapeutical uncertainties. J Thromb Thrombolysis. 2011 Jul;32(1):82-8.

14. Krajčovičová-Kudláčková M, Blažíček P. Elevated homocysteine in vegetarians and lipid peroxidation. Biologia. 2003;58(6):1119-22.

15. McKinley MC, Strain JJ, McPartlin J, Scott JM, McNulty H. Plasma homocysteine is not subject to seasonal variation. Clin Chem. 2001 Aug;47(8):1430-6.

16. Zhang L, Ren A, Li Z, Hao L, Tian Y, Li Z. Folate concentrations and folic acid supplementation among women in their first trimester of pregnancy in a rural area with a high prevalence of neural tube defects in Shanxi, China. Birth Defects Res A Clin Mol Teratol. 2006 Jun;76(6):461-6.

17. Baggott JE, Oster RA, Tamura T. Meta-analysis of cancer risk in folic acid supplementation trials. Cancer Epidemiol. 2012 Feb;36(1):78-81.

18. Pitkin RM. Folate and neural tube defects. Amer J Clin Nutr. 2007;85(1):S285-8.

19. Bailey LB, Gregory JF 3rd. Folate metabolism and requirements. J Nutr. 1999 Apr;129(4):779-82.

20. Frankenburg FR. Folate supplementation: is it safe and effective? J Clin Psychiatry. 2009 May;70(5):767.

21. Morris MS, Jacques PF, Rosenberg IH, Selhub J. Circulating unmetabolized folic acid and 5-methyltetrahydrofolate in relation to anemia, macrocytosis, and cognitive test performance in American seniors. Am J Clin Nutr. 2010 Jun;91(6):1733-44. 